BULLETIN OF THE

AMERICAN MATHEMATICAL SOCIETY

Volume 79, Number 4, July 1973

\title{
AN EXTENSION OF A THEOREM OF HAMADA ON THE CAUCHY PROBLEM WITH SINGULAR DATA ${ }^{1}$
}

BY LESLIE LAMPORT

Communicated by François Trèves, January 8, 1973

Introduction. Hamada [1] proved the following result about the propagation of singularities in the Cauchy problem for an analytic linear partial differential operator. Assume that the initial data are analytic at the point $\mathbf{0}$ except for singularities along a submanifold $T$ of the initial surface containing $\mathbf{0}$. Let $K^{(1)}, \cdots, K^{(m)}$ be the characteristic surfaces of the operator emanating from $T$. Under the assumption that the $K^{(i)}$ have multiplicity one, he showed that the solution of the Cauchy problem is analytic at $\mathbf{0}$ except for logarithmic singularities along the $K^{(i)}$. We extend his result to the case where the $K^{(i)}$ have constant multiplicity.

1. Definitions and theorem. Let $\boldsymbol{C}^{n+1}$ denote the set of $(n+1)$-tuples $\boldsymbol{x}=\left(x^{0}, \ldots, x^{n}\right)$ of complex numbers. Let $S$ be an $n$-dimensional analytic submanifold of $C^{n+1}$, and let $T$ be an $(n-1)$-dimensional analytic submanifold of $S$. Since our results are local, we can assume $S=\{(0$, $\left.\left.x^{1}, \ldots, x^{n}\right) \in C^{n+1}\right\}$ and $T=\left\{\left(0,0, x^{2}, \ldots, x^{n}\right) \in C^{n+1}\right\}$.

Let $D_{i}=\partial / \partial x^{i}, \boldsymbol{D}=\left(D_{0}, \ldots, D_{n}\right)$, and let $a: \boldsymbol{x} \rightarrow a(\boldsymbol{x} ; \boldsymbol{D})$ be an analytic partial differential operator on a neighborhood of 0 in $C^{n+1}$. Let $h(x ; D)$ be the principal part of $a(x ; D)$. We assume that $S$ is not a characteristic surface of $a$ at $\mathbf{0}$, so $h(\mathbf{0} ; 1,0, \ldots, 0) \neq 0$. Let $\boldsymbol{p}=\left(p_{0}, \ldots, p_{n}\right)$ be an $(n+1)$-tuple of formal variables, so $h(x ; p)$ is a homogeneous polynomial in $\boldsymbol{p}$ with analytic coefficients.

We say that the operator $a$ has constant multiplicity at $\mathbf{0}$ in the direction of $T$ if we can factor $h$ as

$$
h(\boldsymbol{x} ; \boldsymbol{p})=\left[h_{1}(\boldsymbol{x} ; \boldsymbol{p})\right]^{k_{1}} \cdots\left[h_{s}(\boldsymbol{x} ; \boldsymbol{p})\right]^{k_{s}}
$$

for all $\boldsymbol{x}$ in a neighborhood of $\mathbf{0}$, where each $h_{i}(\boldsymbol{x} ; \boldsymbol{p})$ is a polynomial in $p$ of degree $m_{i}$ with analytic coefficients, and the $\Sigma m_{i}$ roots of the polynomials $h_{i}(\mathbf{0} ; \tau, 1,0, \ldots, 0)$ in $\tau$ are all distinct. If $s=k_{1}=1$, then $a$ is said to be of multiplicity one at $\mathbf{0}$ in the direction of $T$.

Assume now that $a$ has constant multiplicity at $\mathbf{0}$ in the direction of $T$. It can be shown that we can find $m=\Sigma m_{i}$ analytic characteristic functions $\varphi^{(1)}, \ldots, \varphi^{(m)}$ of $h$ defined in a neighborhood $N$ of $\mathbf{0}$ satisfying:

AMS (MOS) subject classifications (1970). Primary 35A20; Secondary 35C10.

Key words and phrases. Analytic Cauchy problem, characteristic surfaces, constant multiplicity, singularities.

${ }^{1}$ The results described here are contained in the author's $1972 \mathrm{Ph} . \mathrm{D}$. dissertation, written at Brandeis University under the supervision of Professor Richard Palais.

Copyright $(C)$ American Mathematical Society 1973 
1. $h\left(x ; D \varphi^{(i)}(x)\right)=0$ for all $x \in N$.

2. $\varphi^{(i)}\left(0, x^{1}, \ldots, x^{n}\right)=x^{1}$ for all $\left(0, x^{1}, \ldots, x^{n}\right) \in N \cap S$.

3. For each $y \in N \cap S$, the $m$ numbers $D_{0} \varphi^{(i)}(y)$ are distinct.

Note that this implies that the numbers $D_{0} \varphi^{(i)}(y)$ are the distinct roots of the polynomials $h(y ; \tau, 1,0, \ldots, 0)$ for each $y \in N \cap S$. Let $K^{(i)}=$ $\left\{\boldsymbol{x}: \varphi^{(i)}(\boldsymbol{x})=0\right\}$, so each $K^{(i)}$ is a characteristic surface of $a$.

Using these notations, we now state our result.

THEOREM. Let $a, N, S, T, \varphi^{(i)}$ and $K^{(i)}$ be as above. Let $v$ be an analytic function on $N$, and let $w^{j}$ be an analytic function on $N \cap(S-T)$ for $j=0$, $\ldots, r-1$, where $r$ is the degree of the operator a. Then there exists a neighborhood $U$ of $\mathbf{0}$ such that the Cauchy problem

(1) $a(x ; D) u(x)=v(x)$

$\left(D_{0}\right)^{j} u(y)=w^{j}(y), \quad$ for $y \in S, j=0, \ldots, r-1$, has a solution $u$ of the form

$$
u(x)=\sum_{i=1}^{m} F^{(i)}(x)+G^{(i)}(x) \log \left[\varphi^{(i)}(x)\right],
$$

where each $F^{(i)}$ is analytic on $U-K^{(i)}$ and each $G^{(i)}$ is analytic on $U$.

Hamada proved this result when $a$ has multiplicity one. In this case, if each $w^{j}$ has at most a polar singularity along $T$, then each $F^{(i)}$ has at most a polar singularity along $K^{(i)}$. This is false in the general case, as is shown by the solution

$$
u(t, y)=\sum_{k=0}^{\infty}(-1)^{k} \frac{k !}{(2 k+1) !} \frac{t^{2 k+1}}{y^{k+1}}
$$

of the two-dimensional Cauchy problem

$$
\frac{\partial^{2} u}{\partial t^{2}}(t, y)-\frac{\partial u}{\partial y}(t, y)=0, \quad u(0, y)=0, \quad \frac{\partial u}{\partial t}(0, y)=\frac{1}{y} .
$$

2. Method of proof. The problem is easily reduced to solving the Cauchy problem (1) with each $w^{j} \equiv 0$ and $v$ analytic on $N-K^{(1)}$. It can be shown that we may also assume that $h(\boldsymbol{x} ; \boldsymbol{p})=h_{1}(\boldsymbol{x} ; \boldsymbol{p}) \cdots h_{s}(\boldsymbol{x} ; \boldsymbol{p})$, where each $h_{i}$ has multiplicity one in the direction of $T$ and has $\varphi^{(1)}, \ldots, \varphi^{(m)}$ as characteristic functions (so $r=m s$ ).

Let the functions $f_{k}$ be the ones defined by Hamada satisfying $d f_{k} / d t$ $=f_{k-1}$, for all integers $k$, and $f_{0}(t)=\log t$. The first step is to show that there exists a neighborhood $V$ of $\mathbf{0}$ such that if $v$ is of the form

$$
v(x)=\sum_{i=1}^{m} \sum_{k=0}^{\infty} v_{k}^{(i)}(x) f_{k-l}\left[\varphi^{(i)}(x)\right]
$$

with each $v_{k}^{(i)}$ analytic on $V$, then the Cauchy problem 
$h_{i}(\boldsymbol{x} ; \boldsymbol{D}) u(\boldsymbol{x})=v(\boldsymbol{x}), \quad\left(D_{0}\right)^{j} u(\boldsymbol{y})=0, \quad$ for $\boldsymbol{y} \in S, j=0, \ldots, m-1$,

has a formal series solution of the form

$$
u(x)=\sum_{i=1}^{m} \sum_{k=0}^{\infty} u_{k}^{(i)}(x) f_{k-l+m-1}\left[\varphi^{(i)}(x)\right]
$$

with each $u_{k}^{(i)}$ analytic on $V$. Moreover, bounds are obtained for the partial derivatives of the $u_{k}^{(i)}$ in terms of those of the $v_{k}^{(i)}$. This procedure is similar to the one used by Hamada.

Employing this result $s$ times shows that with $v$ given by (2), the Cauchy problem

$h_{1}(\boldsymbol{x} ; \boldsymbol{D}) \cdots h_{s}(\boldsymbol{x} ; \boldsymbol{D}) u(\boldsymbol{x})=v(\boldsymbol{x}),\left(D_{0}\right)^{j} u(\boldsymbol{y})=0$, for $\boldsymbol{y} \in S, j=0, \ldots, r-1$,

has a formal solution

$$
u(x)=\sum_{i=1}^{m} \sum_{k=0}^{\infty} u_{k}^{(i)}(x) f_{k-l+r-s}\left[\varphi^{(i)}(x)\right]
$$

with the $u_{k}^{(i)}$ analytic on $V$. Again, bounds are obtained on the $u_{k}^{(i)}$.

Now we write $a(\boldsymbol{x} ; \boldsymbol{D})=h_{1}(\boldsymbol{x} ; \boldsymbol{D}) \cdots h_{s}(\boldsymbol{x} ; \boldsymbol{D})+b(\boldsymbol{x} ; \boldsymbol{D})$, where the degree of $b$ is less than $r$. Using the above results, we solve the sequence of Cauchy problems

$$
\begin{aligned}
h_{1}(\boldsymbol{x} ; \boldsymbol{D}) \cdots h_{s}(\boldsymbol{x} ; \boldsymbol{D})_{q} u(\boldsymbol{x}) & = \begin{cases}v(\boldsymbol{x}) & \text { if } q=0, \\
-b(\boldsymbol{x} ; \boldsymbol{D})_{q-1} u(\boldsymbol{x}) & \text { if } q>0 .\end{cases} \\
\left(\boldsymbol{D}_{0}\right)_{q}^{j} u(\boldsymbol{y}) & =0, \text { for } \boldsymbol{y} \in S, j=0, \ldots, r-1,
\end{aligned}
$$

to get

$$
{ }_{q} u(x)=\sum_{i=1}^{m} \sum_{k=0}^{\infty}{ }_{q} u_{k}^{(i)}(x) f_{k-l-q(s-1)}\left[\varphi^{(i)}(\boldsymbol{x})\right]
$$

with each ${ }_{q} u_{k}^{(i)}$ analytic on $V$. Then

$$
u(x)=\sum_{q=0}^{\infty} q u(x)
$$

is easily seen to be a formal solution of (1) (with $w^{j} \equiv 0$ ).

Now assume $v(\boldsymbol{x})=v_{l}(\boldsymbol{x}) f_{-l}\left[\varphi^{(1)}(\boldsymbol{x})\right]$, with $v_{l}$ analytic on $V$, and let the corresponding solution (4) be $u_{l}(x)=\sum_{i=1}^{m} u_{l}^{(i)}(x)$. Using the bounds on the ${ }_{q} u_{k}^{(i)}$, we can find a neighborhood $W$ of $\mathbf{0}$ and demonstrate the absolute convergence of the sums (3) and (4) to prove that $u_{l}^{(i)}$ is analytic on $W-K^{(i)}$. Furthermore, we obtain a bound on $u_{l}^{(i)}$ in terms of a bound on $v_{l}$.

Finally, we can write $v(x)=\sum_{l=1}^{\infty} v_{l}(\boldsymbol{x}) f_{-l}\left[\varphi^{(1)}(\boldsymbol{x})\right]$ (plus an analytic term which is handled by the Cauchy-Kowalewski theorem). It can be shown that there is a neighborhood $U$ of 0 such that the sums $u^{(i)}(x)=\sum_{l=1}^{\infty} u_{l}^{(i)}(x)$ 
are absolutely convergent on $U-K^{(i)}$. It is then easily seen that the solution $u(x)=\sum_{i=1}^{m} u^{(i)}(x)$ has the desired form.

3. Further generalizations. It is evident from the proof that the theorem remains valid if $v$ has a singularity along any of the hypersurfaces $K^{(i)}$. The theorem is also true if $v$ has a singularity on any hypersurface $K$ containing $T$ which is not tangent to $S$ or to any $K^{(i)}$ at $\mathbf{0 .}$

By using different choices for the functions $f_{k}$, the result can be extended to the case where the $w^{j}$ are $p$-valued analytic functions on $N \cap(S-T)$-i.e., multiple-valued functions finitely ramified about $T$ and $v$ is a $p$-valued analytic function on $N-K^{(i)}$ or $N-K$. In this case, the $F^{(i)}$ become $p$-valued analytic functions on $U-K^{(i)}$. This result was also obtained by Wagschal [2] when $a$ has multiplicity one.

\section{REFERENCES}

1. Y. Hamada, The singularities of the solutions of the Cauchy problem, Publ. Res. Inst. Math. Sci. 5 (1969), 21-40. MR 40 \# 3056.

2. C. Wagschal, Problème de Cauchy analytique, à données meromorphe, J. Math. Pures Appl. (to appear).

Massachusetts Computer Assoclates, Lakeside Office Park, Wakefield, MassaCHUSETTS 01880 